\title{
Arts, mental distress, mental health functioning \& life satisfaction: fixed-effects analyses of a nationally-representative panel study
}

\author{
Senhu Wang ${ }^{1}$, Hei Wan Mak ${ }^{2}$ and Daisy Fancourt ${ }^{2 *}$ (1)
}

\begin{abstract}
Background: Arts engagement within communities is ubiquitous across cultures globally and previous research has suggested its benefits for mental health and wellbeing. However, it remains unclear whether these benefits are driven by arts engagement itself or by important confounders such as socio-economic status (SES), childhood arts engagement, previous mental health, personality, or self-selection bias. The aim of this study is to use fixed effects models that account for unidentified time-constant confounding measures to examine the longitudinal association between arts (frequency of both arts participation and cultural attendance), mental distress, mental health functioning and life satisfaction.

Methods: Data from 23,660 individuals (with a mean age of 47 years) included in the UK Understanding Society wave 2 (2010-2012) and wave 5 (2013-2015) were analyzed. Aside from controlling for all time-constant variables using fixed-effects models, we additionally adjusted for time-varying demographic factors (e.g. age and marital status), health behaviors and social support variables.

Results: After controlling for all time-constant variables and identified time-varying confounders, frequent arts participation and cultural attendance were associated with lower levels of mental distress and higher levels of life satisfaction, with arts participation additionally associated with better mental health functioning. Health-related and social time-varying factors were shown partly but not wholly to explain the observed associations.

Conclusion: Arts engagement amongst the population as a whole may help enhance positive mental health and life satisfaction, and protect against mental distress. These results are independent of a wide range of time-constant confounding factors.
\end{abstract}

Keywords: Arts engagement, Mental health, Wellbeing, Fixed-effects, Longitudinal study

\section{Background}

Arts engagement within communities is ubiquitous across cultures globally. In the UK alone, there are estimated to be over 40,000 choirs [1], 11,000 amateur orchestras [2], 50,000 amateur arts groups [2], 50,000 book clubs [3], 5000 amateur theatre societies [2], 3000 dance groups [2], 2500 museums [4], and 1300 theatres [5]. Previous research has shown that arts engagement has beneficial

\footnotetext{
* Correspondence: d.fancourt@ucl.ac.uk

${ }^{2}$ Department of Behavioural Science and Health, University College London,

1-19 Torrington Place, London WC1E 7HB, UK

Full list of author information is available at the end of the article
}

effects for both mental health and well-being [6-10]. It has been suggested that this could be due to multiple mechanisms including arts activities enhancing self-identity through the pursuit of skills, stimulation of creativity and self-expression [11], facilitating self-esteem and selfefficacy [12], building an individual's social identity [13], reducing psychological and biological markers of stress [14], providing cognitive stimulation $[15,16]$, enhancing social support $[17,18]$, reducing sedentary behaviours associated with depression [19], and supporting coping skills [20-22].

(c) The Author(s). 2020 Open Access This article is distributed under the terms of the Creative Commons Attribution 4.0 International License (http://creativecommons.org/licenses/by/4.0/), which permits unrestricted use, distribution, and reproduction in any medium, provided you give appropriate credit to the original author(s) and the source, provide a link to the Creative Commons license, and indicate if changes were made. The Creative Commons Public Domain Dedication waiver (http://creativecommons.org/publicdomain/zero/1.0/) applies to the data made available in this article, unless otherwise stated. 
However, a challenge in studies that have been conducted is accounting for confounding factors that could in fact explain any association between arts engagement and both mental health and wellbeing, especially as many of these confounders include complex individual traits and previous life experiences that can be hard to capture fully. For example, arts engagement is socially patterned, with engagement in adult life related to broader social and cultural capital as well as education, income, engagement in childhood and the engagement of parents and wider peer groups [23, 24]. Similarly, arts engagement has been shown to vary based on childhood engagement and the engagement patterns of friends and family as an individual transitions to adulthood [25], and based on previous mental health [26]. Personality too has been shown to affect attitudes towards arts engagement [27]. As these factors are all themselves associated with mental health, it is possible that associations between arts and mental health could in fact be due to individual confounding factors $[28,29]$. This is the case both for intervention studies, for which selfselection bias is likely to predispose individuals with higher levels of cultural capital, greater past experience, better previous mental health and open personality types to take part, and for analyses of observational data. Even longitudinal analyses observational data that adjust for such factors still may not entirely remove their effects [31].

Therefore, to address these issues we analyzed data from a nationally-representative and longitudinal cohort study and applied fixed effects (FE) regression models, which automatically adjust for all time-constant unobserved confounders and help reduce the risk of omitted variable bias, as well as additionally adjusting for identified time-varying confounders.

\section{Method}

\section{Participants}

This study used data from Understanding Society: The UK Household Longitudinal Study (UKHLS), which provides high-quality longitudinal panel data comprising a stratified and clustered General Population Sample of around 40,000 households. These analyses used data from wave 2 (2010-2012) and wave 5 (2013-2015) when questions on arts participation/cultural events were included. Of 54,554 respondents in wave 2, 37,389 were followed up in wave 5, and 25, 051 of these (around 67\%) responded to selfcompletion questionnaires on health and arts. After deleting 1391 cases with missing information (around $5 \%$ ), the final analytic sample includes 23,660 respondents and 47,320 person-wave observations.

\section{Measures}

Arts engagement was measured using 28 separate questions that were categorised into participation in active arts participation ("arts participation") or attending cultural events ("cultural attendance"). Arts participation included dance (including ballet), singing to an audience or rehearsing for a performance (not karaoke), playing a musical instrument, writing music, rehearsing/performing in a play/drama, opera/operetta or musical theatre, taking part in a carnival/street arts event, learning or practising circus skills, painting, drawing, printmaking or sculpting, photography, film or video making as an artistic activity, using a computer to create original artworks or animation, taking part in textile crafts, wood crafts or any other crafts such as embroidery, knitting, reading for pleasure (not newspapers, magazines or comics), writing any stories, plays or poetry, or being a member of a book club where people meet up to discuss and share books.

Cultural attendance included attending a film at a cinema or other venue, an exhibition or collection of art, photography, sculpture or a craft exhibition, an event which included video or electronic art, an event connected with books or writing, street arts or a public art display or installation, a carnival or cultural specific festival, a circus, a play/drama, pantomime or musical, an opera/operetta, a classical music performance, a rock, pop or jazz performance, a ballet, a contemporary dance performance, or an African people's dance or South Asian and Chinese dance. For each question, frequency of arts engagement was measured using five categories for participation in arts participation (never, once/twice per year, once per month, once per week, more than once per week) and four categories for attendance at cultural events (never, once/twice per year, once per month, once per week or more).

Given the well-known distinctions between mental health and multidimensional wellbeing [32], we explored three different outcome variables. Mental distress was measured with GHQ-12 (General Health Questionnaire); a well-validated scale derived from 12 items to measure the levels of respondents' psychiatric illness. Items include depressive and anxiety symptoms, sleeping problems, and overall happiness [33]. UKHLS converts the answers to GHQ-12 questions to a single continuous scale ranging from 0 (the least distressed) to 12 (the most distressed), with a lower score indicating better mental health.

Mental functioning was measured using SF-12 (12Item Short Form Health Survey), a widely used and reliable instrument that measures respondents' general quality of life and focuses both on mental and physical health. It places a particular emphasis on the implications of any problems for ability to function as normal 
in everyday life [34]. The survey contains eight indicators formed of 12 items: physical functioning, role limitations due to physical health problems, bodily pain, general health, vitality, social functioning, role limitations due to emotional problems, and mental health [34]. UKHLS calculates the SF-12 Mental Component Summary (MCS) score by assigning higher weights to mental health related items (the latter six items). The MCS score ranges from 0 (the lowest mental functioning) to 100 (the highest mental functioning).

Subjective wellbeing comprises both affective aspects (such as happiness and pleasure in daily life and being free from negative affect) as well as cognitive-evaluative aspects (such as life satisfaction) [35]. We focused specifically on life satisfaction. This was measured using a single-item "overall, how satisfied are you with your life nowadays?" Responses range from 1 (completely unsatisfied) to 7 (completely satisfied) [36].

We used directed acyclic graphs to identify potential confounding factors that could influence both mental health and arts engagement [37]. As our statistical approach controlled automatically for any timeconstant factors, even if unobserved (see 'Statistics'), we restricted our identification of further confounders to those that vary over time. Identified demographic confounders included age, age squared, marital status (never married, married/cohabited, divorced/separated/ widowed), presence of children in the household (no children, preschool children aged $0-4$, primary school children aged 5-11, middle school children aged 1215), employment status (inactive, unemployed, working class, intermediate class, service class), number of people in the household, logged household income, and data collection wave. In order to ascertain whether individuals who engaged in the arts simply led healthier lifestyles, which contributed to their mental health (perhaps as an underlying function of socio-economic status), we additionally controlled for a wide range of health behaviours which are often associated with mental health [38, 39]. These included self-reported sports activity ranking (from 0 'doing no sport at all' to 10 'very active through sport'), smoking behaviour (current smoker, ever smoked, never smoked), drinking frequency in the last year (from 1 - never drink - to 8 drink every day), and portions of fruits or vegetables eaten per day. We also adjusted for the extent to which health limits moderate activities to try and capture the health selectivity in participating in arts activities or events. Finally, in order to identify whether individuals who engaged in the arts simply had stronger social ties and more frequent social contact, we additionally controlled for family support and friend support measured using a 4-point scale from 1 (not at all/no family or friends) to 4 (a lot) for each of the following 3 questions: family/friends understand the way I feel; I can rely on family/friends; I can talk about my worries with family/friends. Principal component factor analyses were conducted to extract one factor for family support (eigenvalue $=2.35$, variance explained $=78 \%$, alpha $=0.86$ ) and one factor for friend support (eigenvalue $=2.45$, variance explained $=82 \%$, alpha $=0.89$ ) . For more details about the distribution of variables, see Table 1.

\section{Statistics}

Using Stata 14, we performed FE regression analyses; a sophisticated statistical technique commonly used in causal inference research. Compared with ordinary least square regression, which does not distinguish between within- and between-person variation, FE regression only focuses on within-person variation, examining how changes in frequencies of art engagement are linked to changes in mental health within each individual over time [40]. In doing this, FE regression eliminates the confounding effects of all time-constant variables (e.g. gender, ethnicity, social class, personality, previous arts engagement, previous mental health, previous education etc). As such, these factors cannot explain any association found. Further, FE regression considers timevarying confounders at both waves, not just at baseline, capturing their dynamic relationship with the exposure and outcome to better estimate the causal relationship.

We fitted nested models adding covariates stepwise. Model 1 automatically adjusted for time-constant variables. Model 2 controlled for time-varying demographic characteritics and wave. Model 3 additionally controlled for time-varying health behaviors and social support. However, as the factors in Model 3 could be seen to lie on the causal pathway (which would make adjusting for them inappropriate), Model 2 may present more appropriate estimates. We further assessed whether age and gender were moderators through including interaction terms.

Although the panel data only consist of two waves, the key variables in this study such as mental health and frequencies of art engagement had enough within variation (on average $35 \%$ of total variation is from within variation) allowing for accurate estimation of FE regression analysis (Allison, 2009).

Data were strongly balanced. A Hausman test confirmed the selection of a fixed effects over a random effects model. The modified Wald test for group-wise heteroscedasticity was significant so sandwich estimators were applied. Coefficients for all years were not jointly equal to zero, so time-fixed effects were included in the model. Longitudinal weights provided by the UKHLS were used to adjust for the complex survey design, non- 


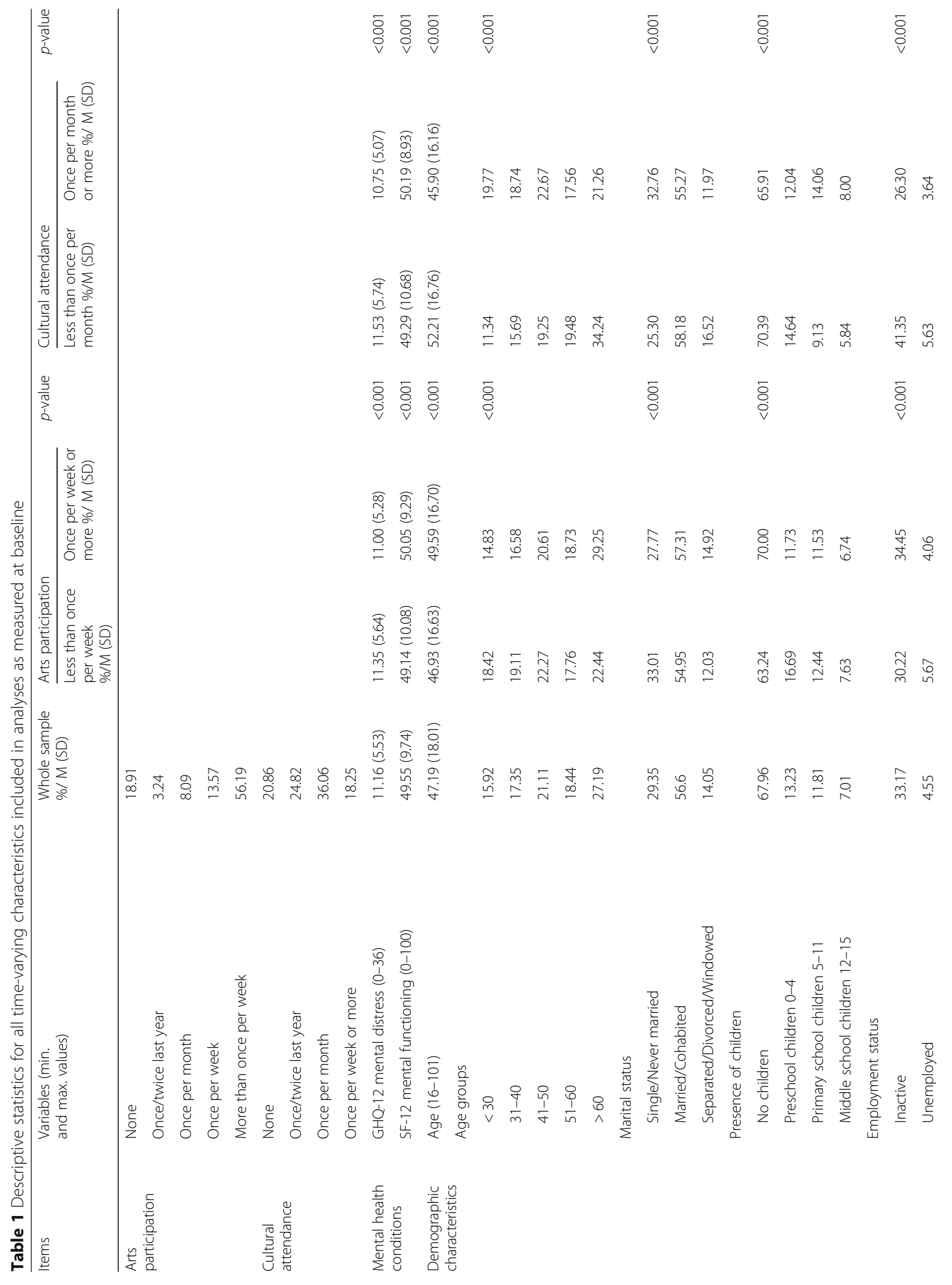




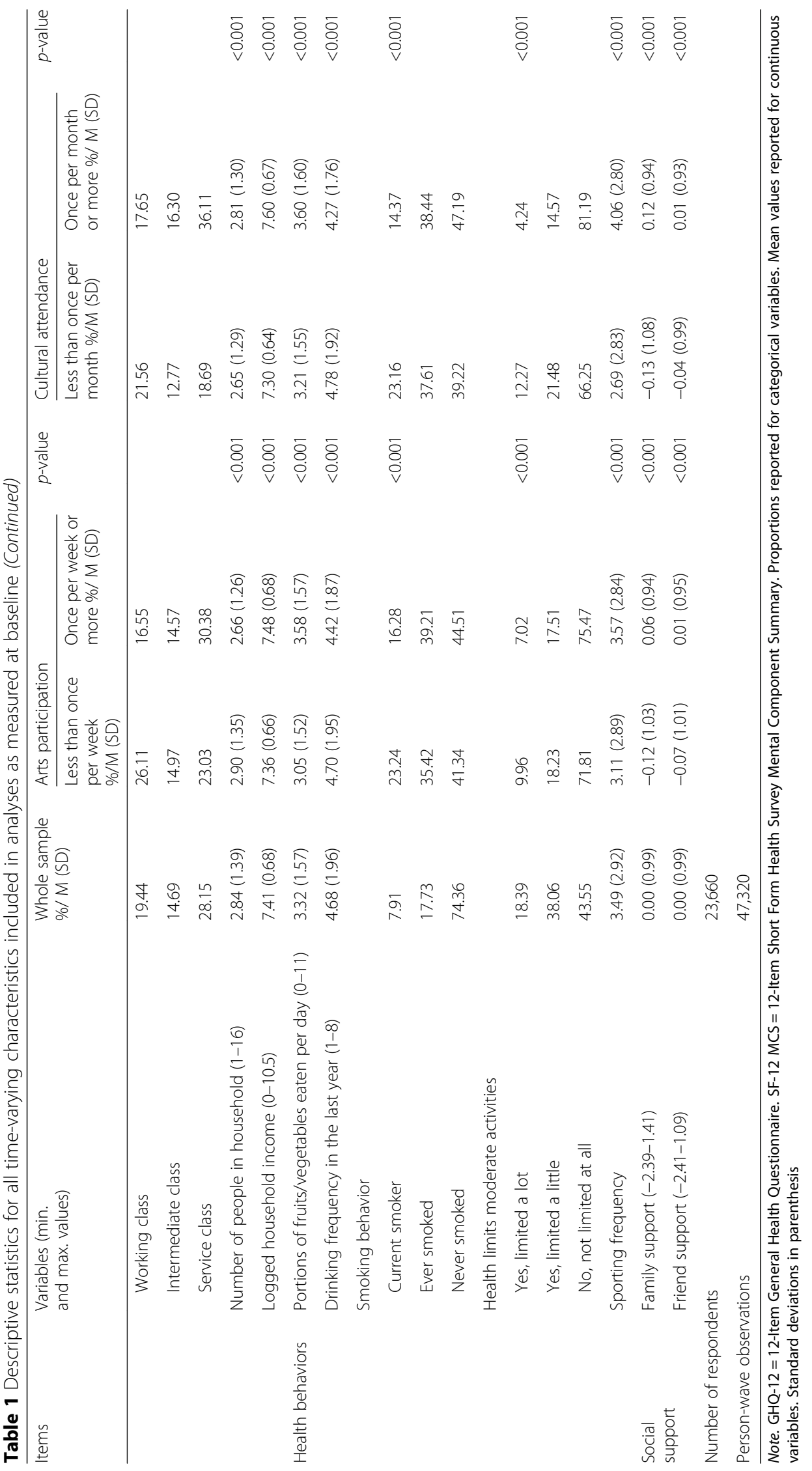


Table 2 Fixed effects models predicting the associations between frequencies of art engagement \& mental health

\begin{tabular}{|c|c|c|c|c|c|c|}
\hline \multirow{2}{*}{ GHQ-12 Mental Distress } & \multicolumn{2}{|c|}{$\begin{array}{l}\text { Model } 1 \\
\text { Coefficients (Cl) }\end{array}$} & \multicolumn{2}{|c|}{$\begin{array}{l}\text { Model } 2 \\
\text { Coefficients (Cl) }\end{array}$} & \multicolumn{2}{|c|}{$\begin{array}{l}\text { Model } 3 \\
\text { Coefficients (Cl) }\end{array}$} \\
\hline & & & & & & \\
\hline \multicolumn{7}{|c|}{ Participation in arts activities (ref $=$ None) } \\
\hline Once/twice per year & -0.35 & $(-0.80,0.11)$ & -0.33 & $(-0.78,0.12)$ & -0.31 & $(-0.75,0.13)$ \\
\hline Once per month & 0.07 & $(-0.29,0.43)$ & 0.04 & $(-0.32,0.39)$ & 0.05 & $(-0.30,0.40)$ \\
\hline Once per week & -0.19 & $(-0.49,0.11)$ & -0.21 & $(-0.51,0.10)$ & -0.21 & $(-0.51,0.09)$ \\
\hline More than once per week & $-0.31^{*}$ & $(-0.60,-0.03)$ & $-0.34^{*}$ & $(-0.62,-0.05)$ & $-0.29^{*}$ & $(-0.56,-0.01)$ \\
\hline Within R-squared & 0.00 & & 0.01 & & 0.03 & \\
\hline \multicolumn{7}{|c|}{ Attendance at cultural events (ref = None) } \\
\hline Once/twice per year & -0.13 & $(-0.40,0.14)$ & -0.14 & $(-0.41,0.13)$ & -0.14 & $(-0.41,0.12)$ \\
\hline Once per month & -0.18 & $(-0.46,0.11)$ & -0.18 & $(-0.46,0.11)$ & -0.14 & $(-0.43,0.14)$ \\
\hline Once per week or more & $-0.51^{* *}$ & $(-0.85,-0.16)$ & $-0.50^{* *}$ & $(-0.85,-0.15)$ & $-0.42^{*}$ & $(-0.76,-0.08$ \\
\hline Within R-squared & 0.00 & & 0.01 & & 0.03 & \\
\hline \multicolumn{7}{|l|}{ SF-12 Mental Functioning } \\
\hline \multicolumn{7}{|c|}{ Participation in arts activities ( $r e f=$ None) } \\
\hline Once/twice per year & 0.71 & $(-0.02,1.45)$ & 0.56 & $(-0.17,1.29)$ & 0.51 & $(-0.21,1.23)$ \\
\hline Once per month & 0.13 & $(-0.47,0.73)$ & 0.11 & $(-0.49,0.70)$ & 0.08 & $(-0.51,0.67)$ \\
\hline Once per week & 0.39 & $(-0.11,0.90)$ & 0.33 & $(-0.17,0.83)$ & 0.33 & $(-0.16,0.82)$ \\
\hline More than once per week & $0.66^{* *}$ & $(0.21,1.12)$ & $0.57^{*}$ & $(0.12,1.02)$ & $0.50^{*}$ & $(0.05,0.95)$ \\
\hline Within R-squared & 0.00 & & 0.02 & & 0.03 & \\
\hline \multicolumn{7}{|c|}{ Attendance at cultural events (ref = None) } \\
\hline Once/twice per year & 0.28 & $(-0.18,0.74)$ & 0.30 & $(-0.16,0.76)$ & 0.31 & $(-0.15,0.76)$ \\
\hline Once per month & 0.25 & $(-0.23,0.73)$ & 0.22 & $(-0.26,0.71)$ & 0.18 & $(-0.30,0.65)$ \\
\hline Once per week or more & $0.63^{*}$ & $(0.05,1.22)$ & 0.56 & $(-0.03,1.15)$ & 0.49 & $(-0.09,1.07)$ \\
\hline Within R-squared & 0.00 & & 0.02 & & 0.03 & \\
\hline
\end{tabular}

Subjective wellbeing: Life satisfaction

Participation in arts activities (ref $=$ None)

\begin{tabular}{|c|c|c|c|c|c|c|}
\hline Once/twice per year & $0.14^{*}$ & $(0.00,0.28)$ & 0.11 & $(-0.03,0.25)$ & 0.11 & $(-0.03,0.25)$ \\
\hline Once per month & 0.08 & $(-0.02,0.18)$ & 0.06 & $(-0.04,0.16)$ & 0.06 & $(-0.04,0.16)$ \\
\hline Once per week & 0.07 & $(-0.02,0.16)$ & 0.05 & $(-0.04,0.14)$ & 0.05 & $(-0.04,0.14)$ \\
\hline More than once per week & $0.12^{* *}$ & $(0.04,0.20)$ & $0.10^{*}$ & $(0.02,0.18)$ & $0.09^{*}$ & $(0.01,0.17)$ \\
\hline Within R-squared & 0.00 & & 0.02 & & 0.03 & \\
\hline \multicolumn{7}{|c|}{ endance at cultural events (ref = None) } \\
\hline Once/twice per year & $0.14^{* * *}$ & $(0.06,0.22)$ & $0.13^{* *}$ & $(0.05,0.21)$ & $0.13^{* *}$ & $(0.05,0.21)$ \\
\hline Once per month & $0.14^{* *}$ & $(0.06,0.23)$ & $0.12^{* *}$ & $(0.04,0.21)$ & $0.11^{*}$ & $(0.03,0.20)$ \\
\hline Once per week or more & $0.21^{* * *}$ & $(0.11,0.32)$ & $0.19^{* * *}$ & $(0.08,0.29)$ & $0.17^{* *}$ & $(0.07,0.27)$ \\
\hline Within R-squared & 0.00 & & 0.02 & & 0.03 & \\
\hline
\end{tabular}

Note: Observations: 47,320. Respondents: 23,660. GHQ-12 = 12-Item General Health Questionnaire. SF-12 MCS = 12-Item Short Form Health Survey. Model $1=$ automatically adjusted for time-constant variables. Model 2 additionally adjusted for demographic factors (age, age squared, marital status, presence of children, employment status, number of people in household, household income and wave). Model 3 additionally adjusted for health-related and social factors (extent to which health limits moderate activities, portions of fruits or vegetables eaten per day, smoking behavior, drinking frequency, sporting frequency, family support and friend support). Confidence intervals in parentheses. ${ }^{* * *} p<0.001,{ }^{* *} p<0.01,{ }^{*} p<0.05$ 
response rate, unequal selection probabilities and nonrandom attrition across waves.

\section{Results}

\section{Decriptive statistics}

Table 1 reports decriptive statistics for the sample as a whole for all time-varying factors, and also compares descriptive characteristics amongst those of frequent and infrequent arts participation and cultural attendance. Overall, the sample had a mean age of 47.2 years $(\mathrm{SD}=$ 18.0 , range $=16-101)$ and was $55.6 \%$ female.

At baseline, people who were frequently engaged in arts had lower levels of mental distress and higher levels of mental functioning and life satisfaction than those who were infrequently engaged as well as better health behaviors, more social support from family and friends, and higher socioeconomic status. Mental distress showed a negative correlation with mental functioning $(\mathrm{r}=-0.73, p<.001)$ and life satisfaction $(r=-0.49$, $p<.001)$, while mental functioning and life satisfaction were positively correlated $(0.47, p<.001)$.

\section{Mental distress}

When adjusting for all identified confounders, significantly lower levels of mental distress were found amongst those who participated in arts activities more than once a week (coef. $-0.29,95 \%$ CI $-0.56,-0.01$ ), or who attended cultural events once a week or more (coef. $-0.42,95 \%$ CI $-0.76,-0.08$ ) (Table 2).

\section{Mental functioning}

When accounting just for time-constant factors, participation in arts activities was associated with significantly higher mental functioning (coef. 0.66, 95\% CI 0.21, 1.22), with this result maintained when considering all identified time-varying confounders (coef. 0.50, 95\% CI 0.05, 0.95) (Table 2). The association between attending cultural events and mental functioning shown when just accounting for time-constant factors (coef. 0.63, 95\% CI $0.05,1.22$ ) was attenuated when controlling for timevarying confounders.

\section{Subjective wellbeing: life satisfaction}

When adjusting for all identified confounders, people who participated in arts activities more than once a week (coef. $0.09,95 \%$ CI $0.01,0.17$ ), or who attended cultural events at least once/twice per year (coef. 0.13, 95\% CI $0.05,0.21$ ) had significantly higher life satisfaction than those who had not participated in these art activities or cultural events (Table 2).

\section{Discussion}

Our study showed a relationship between both frequent arts participation and cultural attendance and lower levels of mental distress and life satisfaction. The results were particularly strong for life satisfaction, for which there were also associations from less frequent cultural attendance. Arts participation was also associated with higher levels of mental functioning. Our results therefore confirm a relationship between arts engagement and multiple different aspects of mental health. This supports previous work using representative samples that had similar findings [4144]. But this finding builds on these previous studies by using a sophisticated statistical technique that shows that although arts engagement is associated with broader aspects of social and cultural capital and socio-economic status (which are themselves associated with health) [28], the relationship is independent of these factors. This finding goes against a finding from a recent study using Swiss data that specifically explored the role of socio-economic status as a confounder, but is supported by another recent study exploring the same question using UK data of older adults [41, 45]. Further, our analyses automatically accounted for other time-constant factors such as personality, previous arts engagement, and previous mental health, suggesting that the associations found in this study could not be explained by any of these factors.

This study is observational and limited by only having data across two waves of UKHLS which do not permit lagged analyses. Therefore, this paper does not attempt to show the direction of the relationship. Instead, it focuses on showing the independence of this relationship from time-constant factors. However, there is a large literature using experimental approaches showing that arts activities can affect mental health [10]. Arts engagement can be considered as a 'complex' or 'multimodal' health-promoting activity in that it combines multiple health-promoting or risk-reducing factors such as gentle physical activity, social interaction, relaxation, emotional expression, and cognitive stimulation [10, 46]. Our analyses could shed further light onto the causal mechanisms that could link arts and mental health as we found a relationship for higher mental health functioning only with arts participation; not cultural attendance. As cultural attendance is associated with improved mental distress and life satisfaction directly but not functioning, this suggests a direct relationship with affective symptoms (such as reductions in negative feelings and stress hormones and enhanced feelings of happiness) but not with an ability to alter psychological or behavioural factors relating to coping with affective symptoms. However, arts participation does show a relationship with mental functioning. As the major distinction between the two types of arts engagement is the participation itself, as multiple other 
elements of the two types of activities (e.g. aesthetic engagement, gentle physical activity, social interaction etc) are consistent, this suggests it is participation that supports coping. Whether there are different causal effects of arts participation and cultural attendance on mental functioning remains to be explored further. But this proposed difference in mechanisms between the two types of engagement is supported by several intervention studies that have identified improvements in aspects of functioning such as self-efficacy, agency and purpose as a result of arts participation [47].

Our study has a number of strengths including its large, representative sample, its longitudinal design, its use of a statistical model that considers both timeconstant and time-varying factors, its rich data on different types of arts engagement, and its comparison of multiple related measures of mental health and wellbeing. However, it is possible that residual confounding for time-varying factors remains. Nevertheless, as all time-constant factors are automatically considered and the data allowed us to include all identified confounders, remaining unobserved heterogeneity should be small. Our analyses also focused on broad categories of arts engagement. Future research may like to consider different types of arts or cultural activities in greater detail and consider how access to activities within communities may affect abilities to engage. Finally, given that the data used in this study was from a national, representative data set and that a large sample size was maintained for the analyses, the generalizability is assumed to be relatively high. However, further work is required to examine whether the findings of this study can be replicated in different settings (such as in data from countries or more specific samples), and whether this finding is common sub-groups, in particular individuals with mental illness.

\section{Conclusions}

Overall, our results demonstrate associations between arts participation, cultural attendance and multiple aspects of mental health and wellbeing. Importantly, we found that such associations are independent of timeconstant socio-demographic, historical or personalitybased factors. Future large-scale studies are encouraged to explore the potential of arts interventions as a public health strategy to help promote both mental health and wellbeing.

\section{Abbreviations}

FE: Fixed effects; GHQ-12: General Health Questionnaire; MCS: Mental Component Summary; SF-12: 12-Item Short Form Health Survey; UKHLS: UK Household Longitudinal Study

Acknowledgements

Not applicable.

\section{Authors' contributions}

SW conducted the data management, data analyses and provided input on the manuscript. HWM and DF assisted with analytical issues, provided input on the analytical scheme and the manuscript. All authors read and approved the final manuscript.

\section{Funding}

DF is supported by the Wellcome Trust [205407/Z/16/Z]. HWM is funded through the AHRC project HEARTS [AH/P005888/1]. The research also received funding from the ESRC project WELLCOMM [ES/T006994/1].

\section{Availability of data and materials}

UKHLS data is available from the UK Data Service https://discover. ukdataservice.ac.uk/catalogue/?sn=6614. Data documentation is available from the Understanding Society website https://www.understandingsociety. ac.uk/documentation.

\section{Ethics approval and consent to participate}

The University of Essex Ethics Committee has approved all data collection on Understanding Society main study and innovation panel waves, including asking consent for all data linkages except to health records. Respondents aged 16 or above provided written consent to participate.

Consent for publication

Not applicable.

\section{Competing interests}

The authors declare that they have no competing interests.

\section{Author details}

${ }^{1}$ Department of Sociology, National University of Singapore, Singapore, Singapore. ${ }^{2}$ Department of Behavioural Science and Health, University College London, 1-19 Torrington Place, London WC1E 7HB, UK.

Received: 12 June 2019 Accepted: 19 December 2019

Published online: 11 February 2020

\section{References}

1. Voices Now: Big Choral Census (UK). https://musiceducationuk.com/ resources/voices-now-big-choral-census-uk/. Accessed 19 Jan 2018.

2. Our Creative Talent - the voluntary and amateur arts in England | CultureHive. http://www.culturehive.co.uk/resources/our-creative-talent-thevoluntary-and-amateur-arts-in-england/. Accessed 19 Jan 2018.

3. Hartley J. Reading groups: Oxford University Press; 2001.

4. FAQs | Museums Association. https://www.museumsassociation.org/about/ frequently-asked-questions. Accessed 19 Jan 2018.

5. How many theatres are there in the UK? Theatres Trust. http://www. theatrestrust.org.uk/discover-theatres/theatre-faqs/167-how-many-theatresare-there-in-the-uk. Accessed 19 Jan 2018.

6. Curtis A, Gibson L, O'Brien M, Roe B. Systematic review of the impact of arts for health activities on health, wellbeing and quality of life of older people living in care homes. Dementia. 2018;17:645-69.

7. Fujiwara D, Kudrna L, Dolan P. Quantifying and valuing the wellbeing impacts of culture and sport. 2014. https://www.gov.uk/government/ publications/quantifying-and-valuing-the-wellbeing-impacts-of-culture-andsport. Accessed 20 May 2015.

8. Gold C, Voracek M, Wigram T. Effects of music therapy for children and adolescents with psychopathology: a meta-analysis. J Child Psychol Psychiatry. 2004;45:1054-63.

9. Zarobe L, Bungay $\mathrm{H}$. The role of arts activities in developing resilience and mental wellbeing in children and young people a rapid review of the literature. Perspect Public Health. 2017;137:337-47.

10. Fancourt $D$, Finn $S$. What is the evidence on the role of the arts in improving health and well-being? A scoping review. Copenhagen: WHO Regional Office for Europe; 2019 (Health Evidence Network (HEN) synthesis report 67).

11. Maslow AH. Toward a psychology of being. Simon and Schuster; 2013

12. Franklin M. Art Therapy and Self-Esteem Art Ther. 1992;9:78-84.

13. Davidson L, Strauss JS. Sense of self in recovery from severe mental illness. Br J Med Psychol. 1992;65(Pt 2):131-45. 
14. Finn S, Fancourt D. The biological impact of listening to music in clinical and nonclinical settings: A systematic review. Prog Brain Res. 2018;237:173 200.

15. Camic PM, Chatterjee HJ. Museums and art galleries as partners for public health interventions. Perspect Public Health. 2013;133:66-71.

16. Wang S, Blazer DG. Depression and cognition in the elderly. Annu Rev Clin Psychol. 2015;11:331-60.

17. Cohen S, Wills TA. Stress, social support, and the buffering hypothesis. Psychol Bull. 1985;98:310-57.

18. Cohen G. Research on creativity and aging: the positive impact of the arts on health and illness. Generations. 2006;30:7-15.

19. Teychenne M, Ball K, Salmon J. Sedentary behavior and depression among adults: a review. Int J Behav Med. 2010;17:246-54.

20. Hutchinson SL, Loy DP, Kleiber DA, Dattilo J. Leisure as a coping resource: variations in coping with traumatic injury and illness. Leis Sci. 2003;25:143-61.

21. Perkins $R$, Yorke $S$, Fancourt D. How group singing facilitates recovery from the symptoms of postnatal depression: a comparative qualitative study. BMC Psychol. 2018:6:41.

22. Perkins R, Ascenso S, Atkins L, Fancourt D, Williamon A. Making music for mental health: how group drumming mediates recovery. Psychol WellBeing. 2016;6:11

23. Blood I, Lomas M, Robinson M. Every child : equality and diversity in arts and culture with, by and for children and young people; 2016.

24. Bourdieu P. The forms of capital. Cult Theory Anthol. 1986;1:81-93.

25. Elsley S, McMellon C. Starting young? Links between childhood and adult participation in culture and science: a literature review; 2010.

26. Jensen A. Mental health recovery and arts engagement. J Ment Health Train Educ Pract. 2018:13:157-66.

27. McManus IC, Furnham A. Aesthetic activities and aesthetic attitudes: influences of education, background and personality on interest and involvement in the arts. Br J Psychol. 2006;97:555-87.

28. Pinxten $\mathbf{W}$, Lievens J. The importance of economic, social and cultura capital in understanding health inequalities: using a Bourdieu-based approach in research on physical and mental health perceptions. Sociol Health IIIn. 2014:36:1095-110.

29. Kendler KS, Gatz M, Gardner CO, Pedersen NL. Personality and major depression: a Swedish longitudinal, Population-Based Twin Study. Arch Gen Psychiatry. 2006;63:1113-20.

30. Wheatley D, Bickerton C. Subjective well-being and engagement in arts, culture and sport. J Cult Econ. 2017;41:23-45.

31. Becher $\mathrm{H}$. The concept of residual confounding in regression models and some applications. Stat Med. 1992;11:1747-58.

32. Keyes CL. Mental illness and/or mental health? Investigating axioms of the complete state model of health. J Consult Clin Psychol. 2005;73:539-48.

33. Goldberg DP. User's guide to the general health questionnaire. Windsor. 1988.

34. Ware J, Kosinski M, Keller S. How to score the SF-12 physical and mental health summary scales. Boston: The Health Institute, New England Medical Center; 1998.

35. Keyes CLM, Shmotkin D, Ryff CD. Optimizing well-being: the empirical encounter of two traditions. J Pers Soc Psychol. 2002:82:1007-22.

36. Dolan P, Layard R, Metcalfe R. Measuring subjective well-being for public policy; 2011.

37. Sauer B, VanderWeele TJ. Use of directed acyclic graphs. In: Developing a protocol for observational comparative effectiveness research: a user's guide. Agency for Healthcare Research and Quality (US); 2013

38. Paluska SA, Schwenk TL. Phsycial activity and mental health. Sports Med 2000;29(3):167-80.

39. Stranges S, Samaraweera PC, Taggart F, Kandala N, Stewart-Brown S. Major health-related behaviours and mental well-being in the general population: the health survey for England. BMJ Open. 2014;4:e005878.

40. Allison PD. Fixed effects regression models. Thousand Oaks: SAGE Publications, Inc; 2009

41. Fancourt D, Steptoe A. Cultural engagement and mental health: Does socio-economic status explain the association? Soc Sci Med. 2019;236: 112425

42. Fancourt D, Steptoe A. Community group membership and multidimensional subjective well-being in older age. J Epidemiol Community Health. 2018;72(5):376-382.
43. Tymoszuk U, Perkins R, Spiro N, Williamon A, Fancourt D. Longitudinal associations between short-term, repeated, and sustained arts engagement and well-being outcomes in older adults. The Journals of Gerontology: Series B. 2019.

44. Wheatley D, Bickerton C. Subjective well-being and engagement in arts, culture and sport. Journal of cultural economics. 2017;41(1):23-45.

45. Węziak-Białowolska D. Attendance of cultural events and involvement with the arts -impact evaluation on health and well-being from a Swiss household panel survey. Public health. 2016:139:161-169.

46. Craig P, Rahm-Hallberg I, Britten N, Borglin G, Meyer G, Köpke S, et al. Researching complex interventions in health: the state of the art. BMC Health Serv Res. 2016;16:101

47. Ascenso S, Perkins R, Atkins L, Fancourt D, Williamon A. Promoting wellbeing through group drumming with mental health service users and their carers. Int J Qual Stud Health Well-Being. 2018;13:1.

\section{Publisher's Note}

Springer Nature remains neutral with regard to jurisdictional claims in published maps and institutional affiliations.

Ready to submit your research? Choose BMC and benefit from:

- fast, convenient online submission

- thorough peer review by experienced researchers in your field

- rapid publication on acceptance

- support for research data, including large and complex data types

- gold Open Access which fosters wider collaboration and increased citations

- maximum visibility for your research: over $100 \mathrm{M}$ website views per year

At BMC, research is always in progress.

Learn more biomedcentral.com/submissions 